\title{
Emissivity Profiles and Radiation Losses in RFX
}

\author{
L. Marrelli, P. Martin ${ }^{1}$, A. Murari ${ }^{2}$, G. Spizzo \\ Consorzio RFX, Padova, Italy \\ ${ }^{1}$ Dipartimento di Fisica, Università di Padova, Padova, Italy \\ ${ }^{2}$ Istituto Gas Ionizzati del CNR, Padova, Italy
}

\begin{abstract}
.
The radiation emitted by RFX plasmas has been investigated using a 8 chord-bolometric camera, whose detectors have been calibrated absolutely. From the experimental data the emissivity profiles have been reconstructed by means of a generalized tomography reconstruction algorithm. This analysis confirms that the emitted radiation in RFX is systematically concentrated at the edge. The emitted power dependence on the plasma density shows that the radiation increases approaching the high density regime, but it rarely goes beyond $30 \%$ of the input power for stationary discharges. This behaviour is strongly dependent on the concentration of impurities, but in any case in RFX there is no evidence of disruptions. A simple local energy balance allows to obtain a preliminary evaluation of the radial heat flux profile.
\end{abstract}

Submitted to: Nuclear Fusion 


\section{Introduction}

The measurement of the power lost by radiation and of the emissivity profiles is crucial for the understanding of the physical properties of a magnetically confined thermonuclear plasma and of its reactor relevance. As far as the Reversed Field Pinch (RFP) magnetic configuration is concerned, in the past this topic has been investigated on various machines, but only with a limited spatial and time resolution [1, 2]. In particular no detailed and systematic analysis of the total radiation emissivity profiles has been reported up to now for a RFP.

To perform these measurements in RFX [3], the highest current RFP experiment nowadays in operation (major radius $R=2 \mathrm{~m}$, minor radius $a=0.457 \mathrm{~m}$, target plasma current $I=2 \mathrm{MA}$ ), a 8 chord - pinhole camera [4], housing miniaturised metal foil bolometers [5], was installed. These detectors, which, thank to their accuracy and reliability, are becoming a standard for total radiation measurements in fusion experiments and are used in the major tokamak and stellarator experiments, such as Tore-Supra [6], JET [7], Asdex-U [8] and FTU [9], were calibrated absolutely in situ, whereas the nominal gain of the amplifiers was doublechecked on the bench before installation, in order to ensure the precision of the measurements. The errors in the determination of the calibration constants and of the electronic gain and the usual level of noise superimposed to the signal can be shown to give a relative uncertainty in the radiated power measurement of the order of $\pm 10 \%$ [10].

The measurements performed with this diagnostic are therefore accurate enough to allow for a sistematic investigation of total radiation emissivity profiles in RFP plasmas and for a thorough analysis of the radiation losses as a function of electron density, in particular in the high density regime.

This paper contains a survey of the steady state emissivity profiles, measured in various operative conditions, during all the essential phases of the 1994/1995 RFX experimental campaign [11], which was dedicated to the investigation of two different current ranges: one between 0.5 and $0.7 \mathrm{MA}$ and one between 0.8 and $0.95 \mathrm{MA}$. The plasma electron density $n_{e}$ was varied in the range between $2 \cdot 10^{19}$ and $1.2 \cdot 10^{20} \mathrm{~m}^{-3}$, whereas the on-axis electron temperature $T_{e}(0)$ was tipically between 200 and $500 \mathrm{eV}$. Typical waveforms of plasma current $I$, electron density $n_{e}$, applied toroidal loop voltage $V_{T}(a)$ and total radiated power $P_{r a d}$ are shown in Fig. 1 for a typical $0.8 \mathrm{MA}$ discharge.

The paper is organised as follows: in Section 2 a short description of the inversion algorithm used to analyse the experimental data is provided, the typical emissivity profiles are presented and a detailed review of the experimental phenomenology is given. Discussions and comments, in particular on global and local power balances, constitute the subject of Section 3.

\section{Inversion algorithm and experimental results}

In the typical arrangement of a pinhole camera an array of detectors views the plasma through a small pinhole aperture. Then each detector identifies, together with the pinhole, a line of sight $L$, characterised by an impact parameter $p$ and a chord angle $\phi$, which are defined respectively as the distance of the line of sight from the center of the plasma cross section and the angle which the normal to the line of sight subtends with the equatorial plane. An account of the values of the impact parameters and chord angles is given in Table I. 


\begin{tabular}{||c|c|c||}
\hline chord number & angle (degrees) & $p / a$ \\
\hline 1 & 34.7 & 0.66 \\
\hline 2 & 26.2 & 0.51 \\
\hline 3 & 16.3 & 0.32 \\
\hline 4 & 5.3 & 0.11 \\
\hline 5 & 174.7 & -0.11 \\
\hline 6 & 163.7 & -0.32 \\
\hline 7 & 153.8 & -0.51 \\
\hline 8 & 145.3 & -0.66 \\
\hline
\end{tabular}

Table I: Chord angles and impact parameters (normalised to the torus minor radius a) for the RFX 8-chord bolometric diagnostic

If the emissivity does not vary in the directions perpendicular to the line of sight, the power impinging on the detector is proportional through geometric factors to the brightness $f(p, \phi)$ (in $\mathrm{W} \mathrm{m}^{-2}$ ), which corresponds to the line integral of the emissivity $\mathcal{E}(r, \theta)\left(\mathrm{W} \mathrm{m}^{-3}\right)$ along its line of sight:

$$
f(p, \phi)=\int_{L(p, \phi)} \mathcal{E}(r, \theta) \mathrm{d} \ell
$$

where $r, \theta$ are the usual polar coordinates over the poloidal cross section. Emissivity distributions can be obtained from the brigthness measurements by means of the inversion of Equation 1, which can be performed with a variety of techniques (see $[12,13]$, and the references therein quoted, for some examples).

Given the limited number of brightness measurements available in the aforementioned pinhole camera and the topology of the lines of sight, arranged in a fan-like geometry, emissivity profiles of the form

$$
\mathcal{E}(r, \theta)=\mathcal{E}_{0}(r)+\mathcal{E}_{1}(r) \cos \theta
$$

have been reconstructed by means of a generalised inversion algorithm; the geometrical lay-out of the chords allows in fact to resolve only the $m=0$ and $m=1$ (cosinusoidal) poloidal harmonics. For $\mathcal{E}_{0}(r)$ and $\mathcal{E}_{1}(r)$ linear piecewise functions (see Fig. 2) have been chosen, which depend on 4 parameters: the maximum of the cosinusoidal component $\mathcal{E}_{1, \max }$, the maximum of the $m=0$ component $\mathcal{E}_{0, \max }$, the value at the plasma centre $(r=0)$ of the $m=0$ component $\mathcal{E}_{0,0}$ and the radial coordinate $\alpha$ of the knee. Moreover, $\mathcal{E}(r)$ is constrained to be zero at the wall $(r=a)$. These parameters are determined by minimising the difference between the experimental brightness measurements and the values analytically computed on the basis of the model.

The shape of the emissivity function $\mathcal{E}(r, \theta)$ was suggested by preliminary indications that the total radiation in RFX usually comes from the outer region of the plasma cross-section; this hypothesis has then been confirmed by the results of the fit, as shown in Fig.3, where the experimental brightness measurements and the reconstructed profiles are reported for three different values $\left(_{h}=1.12,0.02,-0.04\right.$ $\mathrm{cm}$ ) of RFX plasma horizontal magnetic shift (which typically ranges between -2 and $+4 \mathrm{~cm}$ ). Each profile corresponds to a $10 \mathrm{~ms}$ time-average during the current flat-top. The results in this figure confirm that the model is suitable to reproduce the observed macroscopical displacement of the plasma column. 
From the reconstructed emissivity $\mathcal{E}(r, \theta)$ the normalized horizontal position, $x_{G} / a$, of the total radiation "centre of mass", defined as

$$
x_{G} / a=\frac{1}{2 a} \frac{\int_{0}^{a} r^{2} \mathcal{E}_{1}(r) \mathrm{d} r}{\int_{0}^{a} r \mathcal{E}_{0}(r) \mathrm{d} r}
$$

can be determined. As shown in Fig. 4, this quantity proves to be well correlated with the plasma horizontal magnetic shift, not only contributing to reinforce the confidence in the model chosen for the inversion algorithm, but also confirming that appreciable outward displacements of the plasma column, and the ensuing enhanced plasma-wall interactions, correspond to higher radiation in the interacting edge region.

With regard to the experimental conditions, care has been taken to exclude from the analysis those discharges which present toroidally localized strong disturbances of the equilibrium magnetic field, normally interpreted as "locked modes" [14, 15], in proximity of the bolometric camera. These locked modes proved indeed to cause localised phenomena of increased radiation which often have a spatial extention too small to be resolved by the camera and are not representative of the plasma average behaviour. Finally, the normalized plasma toroidal current derivative, defined as $\frac{1}{I} \frac{\mathrm{d} I}{\mathrm{~d} t}$, has been chosen as a typical parameter, in order to distinguish discharges with a rapidly decaying current from those with a better flat-top: values of this parameter less than $-2 \mathrm{~s}^{-1}$ indicate a decaying current, while the interval between -2 and $2 \mathrm{~s}^{-1}$ is typical of discharges for which a good flat-top has been achieved (the typical flat-top duration in RFX ranges between 0.02 and $0.08 \mathrm{~s}$ ).

Irrespective of the detailed shape of the emissivity profiles, the total emitted power increases sharply with the plasma density. This fact is evident from Fig.5, which shows $P_{r a d}$ versus $I / N$, for high current discharges (from 0.8 to $0.95 \mathrm{MA}$ ) whose density was varied in a wider range. $P_{\text {rad }}$ is calculated integrating the reconstructed emissivity $\mathcal{E}(r, \theta)$ over the entire volume

$$
P_{\text {rad }}=2 \pi R \int_{0}^{a} r \mathrm{~d} r \int_{0}^{2 \pi} \mathrm{d} \theta \mathcal{E}(r, \theta)
$$

where $R$ is the torus major radius, and $I / N$, a parameter often used in the analysis of RFP plasmas, is defined as the total plasma toroidal current divided by the linear electron density $\left(N=\int_{0}^{a} 2 \pi r n_{e}(r) \mathrm{d} r\right)$.

In the high density regime (low $I / N$ ), the ohmic input power $P_{\text {ohm }}$ (in RFX, as in all RFPs, there is no auxiliary heating) rises as well, but the relative importance of the radiative loss channel continues to grow. This tendency is confirmed in Fig.6, where the ratio $\gamma=P_{\text {rad }} / P_{o h m}$ is plotted versus $I / N$, for the same set of discharges of Fig. 5. As $I / N$ decreases toward its lower limit $\left(I / N \approx 1.8 \cdot 10^{-14} \mathrm{~A} \mathrm{~m}\right.$ for this set of RFX data), the spread of the values of $\gamma$ becomes wider but on average the fraction of the input power which is radiated is larger (up to $\approx 30 \%$ ).

The dependence of the total emitted radiation on plasma purity has been confirmed by comparing the $\gamma$ ratio for reproducible low current discharges (from 0.5 to $0.7 \mathrm{MA}$ ) at various densities before and after the deposition of a thin film containing Boron [16] on the graphite first wall. The boronization was performed with a radio-frequency assisted Glow Discharge Cleaning (GDC) in a throughflow of a mixture of He and Trimethylboron. After wall conditioning, the plasma purity was improved: in particular the concentrations of oxigen and carbon, relative to the electron density, decreased from $1-2$ to $0.2-0.3 \%$ and from $1-1.5$ to $0.7 \%$ respectively [16]. The resulting plasma effective charge $Z_{\text {eff }}$ decreased from about 2.5 to 1.5 and a 
reduction of the total emitted radiation was observed. The experimentally measured $\gamma$ values were therefore lower for similar levels of $I / N$ as shown in Fig. 7 .

\section{Discussion and conclusions}

\subsection{Global power balance}

The measurements presented in this paper allowed us to quantify the contribution of the total radiation losses to the global power balance. The steady-state global power balance in RFX can be cast in the form:

$$
P_{\text {ohm }}=P_{\text {rad }}+P_{c x}+P_{\text {trans }, c f}+P_{\text {trans }, e e}+P_{l o c}
$$

where $P_{o h m}$ is the total input power, $P_{c x}$ represents losses due to charge exchange, $P_{\text {trans }, c f}$ and $P_{\text {trans,ee }}$ account for losses due to cross field transport and to direct flow of superthermal electrons to the wall respectively, and $P_{l o c}$ takes into account additional localised losses due to locked modes. Charge exchange losses are typically $3-5 \%$ of the total input power at lower densities (high values of $I / N$ ) and decrease at higher densities [17]. As radiation losses rarely exceeds 30\% (Fig.4) of total input power, we conclude that the main loss channel in RFX seems to be transport, even in the high density regimes, characterized by the best confinement properties. Therefore, an experimental exploration of high density regimes and an understanding of transport mechanisms are crucial issues for the future RFP research.

With regard to the high density regimes, the total radiation emitted from RFX plasmas increases decidedly in a barrier-like fashion at low levels of the $I / N$ parameter, as shown in Fig. 5. During the operations with boronized walls, a consistent reduction of $Z_{\text {eff }}$ and a concomitant decrease of radiated power for the same values of $I / N$ were observed. Moreover, it was possible to operate at higher densities, i.e., lower valkues of the $I / N$ parameter. It is still not clear whether this trend of $P_{r a d}$ can be associated with a radiation dominated density limit of the type described in [18], where discharges with almost $100 \%$ of the input power lost by radiation were reported. Indeed, as it is evident in Fig. 6, even at the lower $I / N$ values the $\gamma$ ratio rarely exceeds 0.3 , at least for discharges which show a good flat top of the plasma current. The regime of low $I / N$, where the best confinement performances were obtained [11, 15], is therefore not strictly the one typical of radiation dominated discharges. Moreover, it is indeed worth mentioning than in RFX a further decrease in the $I / N$ parameter seems to be prevented more by operative limitations than by plasma behaviour, since at high density the loop voltage increases dramatically. In any case, no evidence of disruptions, as those associated with the density limit in tokamaks [19,20], was observed.

\subsection{Local power balance}

The emissivity profiles typical of RFX plasmas present a remarkable peak in the edge region. This is consistent with the results of the spectroscopic investigations [21] of the impurity content of the plasma and with the temperatures normally measured at the edge. Indeed the RFX first wall is made of graphite tiles and therefore the most important impurities are Carbon and Oxigen. Their lower ionization states, which are the most radiative, are normally concentrated in the edge region, where the temperature is low (around $15 \mathrm{eV},[22]$ ). The radiative edge region is highly asymmetric, as the $m=1$ component amplitude is comparable to the $m=0$ one for optimally centered discharges (horizontal shift of about $1 \mathrm{~cm}$ ), as can be seen in Fig. 
3b. This sort of external radiative layer extends over the last $30 \%$ of the radius and normally accounts for more than $90 \%$ of the total radiated power.

This experimental evidence seems to confirm the hypothesis that the interior of RFX plasmas are dominated by transport, since the radiation losses are there really low. At the plasma periphery, on the contrary, the contribution of radiation to the power losses can be significant.

At this stage, the available knowledge of the emissivity profiles allows the study of the steady-state local power balance, on the basis of the equation:

$$
\nabla \cdot \mathbf{q}(r)=\Omega(r)-\mathcal{E}(r)
$$

where $\Omega(r)$ is the local ohmic input power dissipation and $\mathbf{q}$ is the heat flux. Integrating over the radius the difference $\Omega(r)-\mathcal{E}(r)$, a rough estimate of the heat flux perpendicular to magnetic surfaces $q_{\perp}$, in steady-state conditions, can be calculated:

$$
q_{\perp}(r)=\frac{1}{r} \int_{0}^{r}\left[\Omega\left(r^{\prime}\right)-\mathcal{E}\left(r^{\prime}\right)\right] r^{\prime} \mathrm{d} r^{\prime}
$$

This heat flux estimate does not depend on assumptions on the scrape off layer geometry as in [23] and is not affected by poloidal asymmetries [17]. The power dissipation term is derived from helicity balance considerations [24]:

$$
\Omega(r)=\frac{I}{\Phi} \eta(r) \mathbf{j}(r) \cdot \mathbf{B}(r)
$$

where $\eta(r)$ is an effective Spitzer-like resistivity given by $\eta(r)[o h m / m]=5.26$. $10^{-5} \log \Lambda Z_{k}^{*} T_{e}(r)_{[e V]}^{-3 / 2}$. The parameter $Z_{k}^{*}$ is determined by matching the volume integral of Equation 8 to the input power determined by integrating the Poynting vector. The current and magnetic field profiles, used in Equation 8, are not directly measured in RFX and are therefore obtained by integrating the equations of the $\mu \& \mathrm{p}$ model [25], with boundary conditions derived from magnetic external measurements. The temperature profiles are fitted by

$$
T_{e}(r)=T_{e}(a)+\left[T_{e}(0)-T_{e}(a)\right]\left[1-\left(\frac{r}{a}\right)^{\alpha_{T}}\right]
$$

where $T_{e}(a)$ is assumed to be equal to $15 \mathrm{eV}$, according to edge measurements [22], and $\alpha_{T}$ is an exponent whose value reasonably ranges in RFX between 3 (peaked profile, as in [23]) and 7 (a flatter profile). This should be considered with some caution, since remarkable uncertainties remain about the actual power dissipation profiles which critically depend on temperature profiles, which are not well diagnosed in the edge region, and on magnetic profiles that are not measured inside the plasma.

Fig. 8a shows the emissivity and local dissipation for RFX shot 4046 (time $30 \mathrm{~ms}$ ), where calculations made assuming three different temperature profiles are presented. The perpendicular heat fluxes, obtained in the same conditions, are shown in Fig. 8b.

The total radiation losses seem to be significant in edge heat flux reduction if the local power deposition is concentrated in the core, as is the case when the temperature porfiles are flatter.

Future investigations on temperature profiles in the edge region will allow us to better inspect the role of radiation in wall load reduction.

More details about bolometric emissivity profiles will be revealed by a new tomographic diagnostic, recently installed on RFX and now in operation.

Systematic impurity injection tests have already been planned, in order to investigate whether a highly emitting layer can be created at the plasma edge. In addition to reducing heat fluxes striking the first wall, theese tests could help to further clarify the pysics of transport. 


\section{Acknowledgements}

The authors would like to acknowledge the contribution of Marco Cecconello to the theoretical investigation of the calibration of the sensors and to the determination of the experimental errors associated with the measurements.

\section{References}

[1] Hokin S et al. 1993 Proceedings of the 20 $0^{\text {th }}$ EPS Conference on Controlled Fusion and Plasma Physics vol 17C Part II (Lisboa, Portugal) p 475

[2] Costa S, R De Angelis, Ortolani S and Puiatti M E 1982 Nucl. Fusion 221301

[3] Fellin R, Kusstatscher P and Rostagni G 1995 Fus. Eng. Des. 25315

[4] Murari A, Mast K F, D’Ambra L et al. 1995 Rev. Sci. Instrum. 66665

[5] Mast F and E R Müller 1984 J. Appl. Phys. 557

[6] Mast K F, Vallet J C et al. 1991 Rev. Sci. Instrum. 62744

[7] Mast K F et al. 1985 Rev. Sci. Instrum. 56969

[8] Andelfinger C et al. 1988 Proceedings of the 15th Symposium on Fusion Technology vol 1 (Utrecht, The Nederlands) p 374

[9] Apruzzese G et al. 1994 Proceedings of the 21 $1^{\text {st }}$ EPS Conference on Controlled Fusion and Plasma Physics vol 2 (Montpellier, France) p 802

[10] MCecconello et al. 1998 Meas. Sci. Technol. 9 579-584

[11] Antoni V et al. 1994 Proceedings of the 15 th IAEA Conference on Plasma Physics and Controlled Nuclear Fusion Research vol 2 (Seville, Spain) p 405

[12] Granetz R S and Smeulders P 1988 Nucl. Fusion 28457

[13] Sabatier P C, GTHerman et al. 1987 Basic Methods of Tomography and Inverse Problems (Adam Hilger)

[14] Antoni V, Bellina F, Bolzonella $\mathrm{T}$ et al. 1995 Proceedings of the 22 ${ }^{\text {nd }}$ EPS Conference on Controlled Fusion and Plasma Physics vol 19C Part IV (Bournemouth, UK) p 181

[15] Antoni V, Buffa A et al. Proceedings of the $16^{\text {th }}$ IAEA Conference on Plasma Physics and Controlled Nuclear Fusion Research (CN 64, DP 25, Vienna, 1996 (to be published))

[16] Sonato P et al. 1996 J. Nucl. Mater. 227 259-265

[17] Buffa A, Antoni V et al. 1994 Proceedings of the 21 $1^{\text {st }}$ EPS Conference on Controlled Fusion and Plasma Physics (Montpellier, France) p 462

[18] Ortolani S and Rostagni G 1983 Nuclear Instrum. Methods 20735

[19] Murakami M, Callen J D, Berry L A et al. 1976 Nucl. Fusion 16347

[20] Greenwald M, Terry J L and Wolfe S M 1988 Nucl. Fusion 282199

[21] Valisa M, Carraro L, Casarotto E et al. 1994 Proceedings of the 21 ${ }^{\text {st }}$ EPS Conference on Controlled Fusion and Plasma Physics (Montpellier, France) p 466

[22] Antoni V, Martines E, Bagatin M, Desideri D and Serianni G 1996 Nucl. Fusion 36 435-442

[23] Antoni V, Apolloni L, Bagatin M et al. 1993 Plasma Phys. Control. Fusion 35 B333

[24] Schoenberg K F, Moses R W and Hagenson R L 1984 Phys. Fluids 271671

[25] Ortolani S 1983 Proceedings of the International School of Plasma Physics, Course on Mirrorbased and Field-reversed Approaches to Magnetic Fusion, Varenna, Italy vol 2 ed Leotta G G et al. (Città di Castello: Franchi) p 513 


\section{Figure captions}

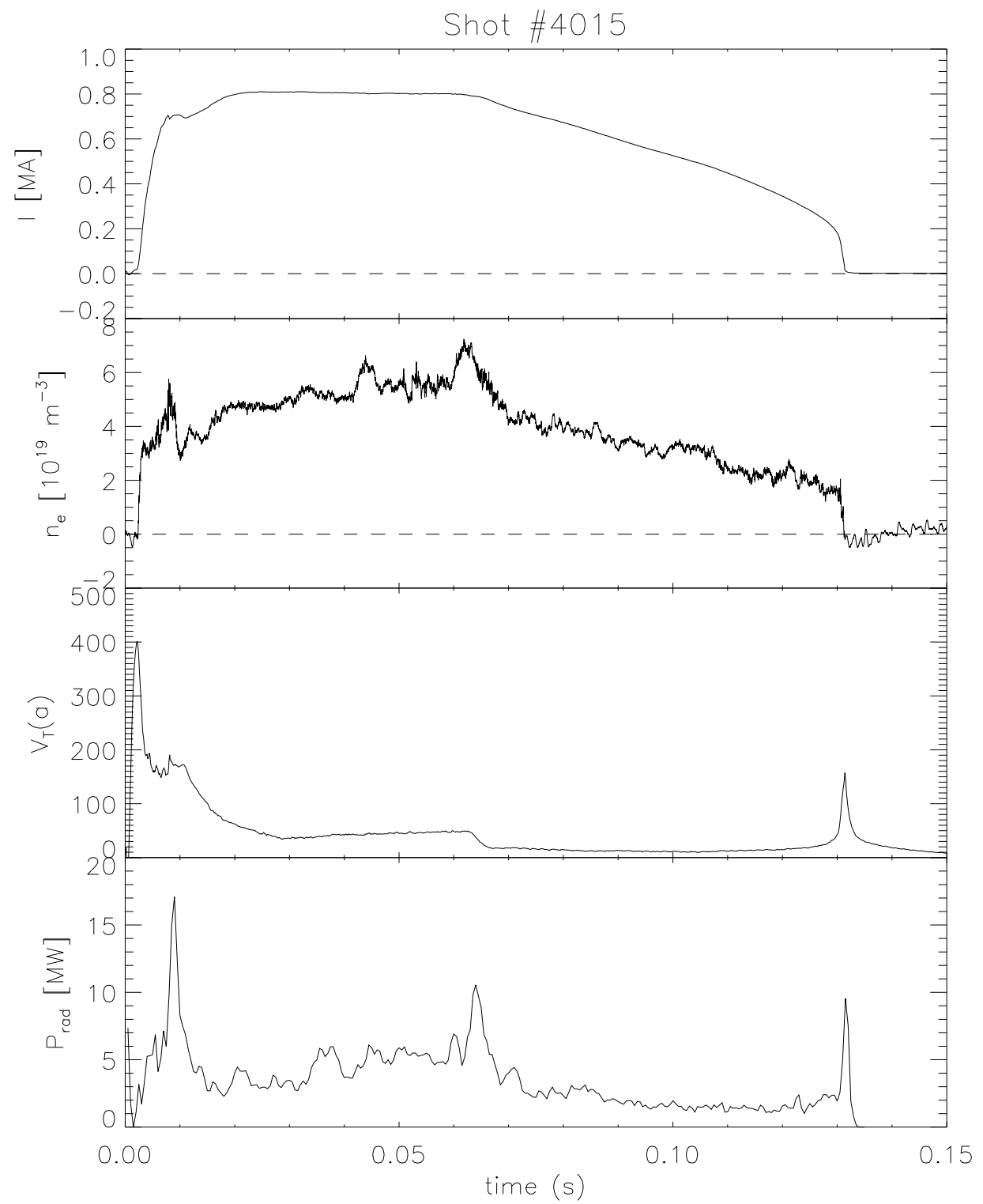

Figure 1: Waveforms of plasma current $I$, electron density $n_{e}$, toroidal loop voltage $V_{T}(a)$ and radiated power $P_{\text {rad }}$ for a typical $800 \mathrm{kA}$ discharge. 

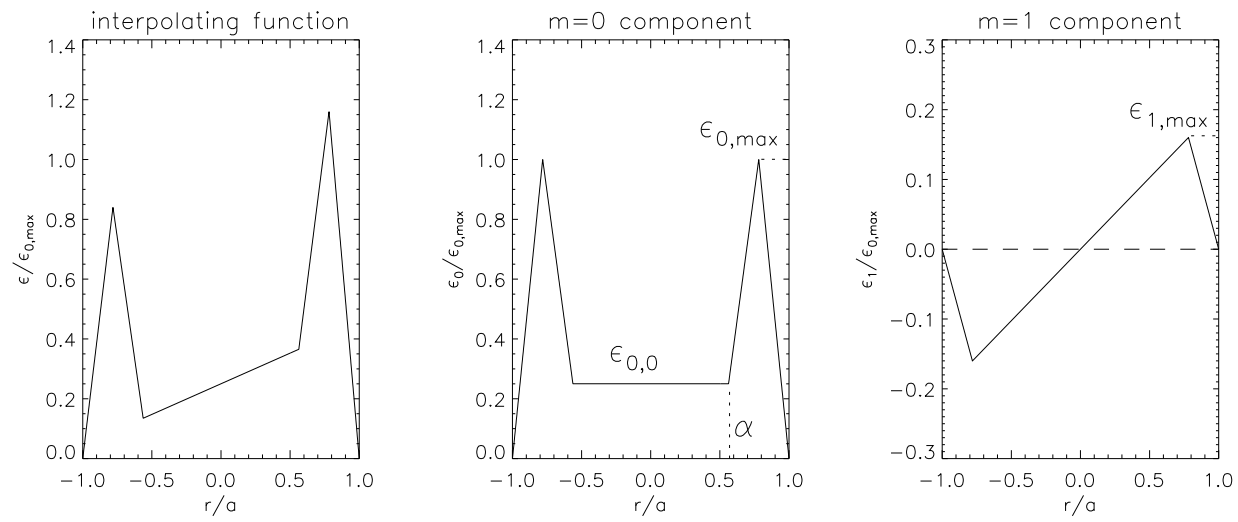

Figure 2: Example of the function used to reconstruct the emissivity profiles, showing the 4 free parameters. 

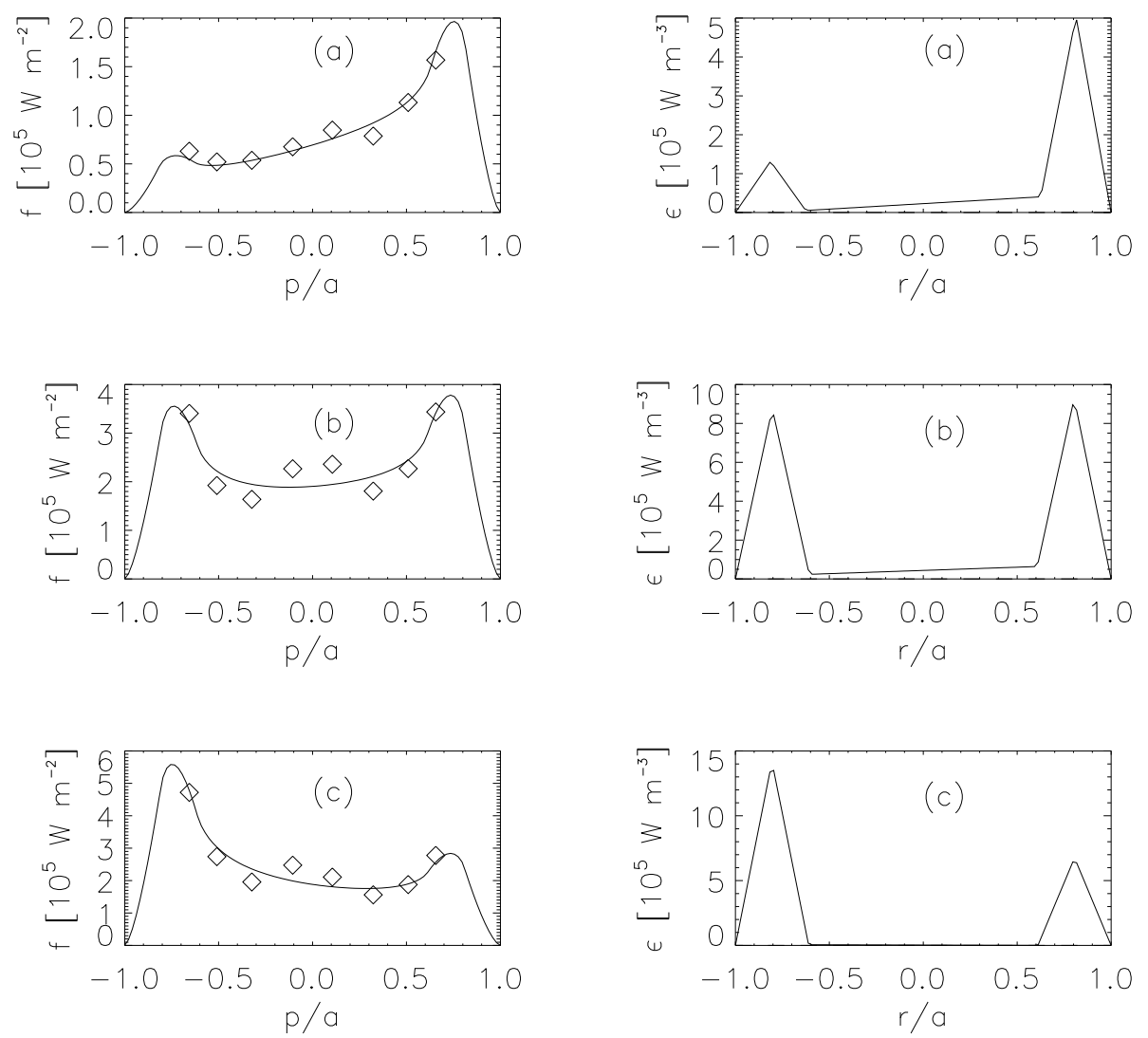

Figure 3: a,c,e) Experimental brightness measurements (diamonds) compared to the fit (solid line) for three shots chaaracterized by different values of magnetic horizontal shift $\Delta_{h}=1.12 \mathrm{~cm}, 0.02 \mathrm{~cm},-0.4 \mathrm{~cm}$, respectively; b,d,f) reconstructed emissivity profiles for the same shots of a,c,e. 


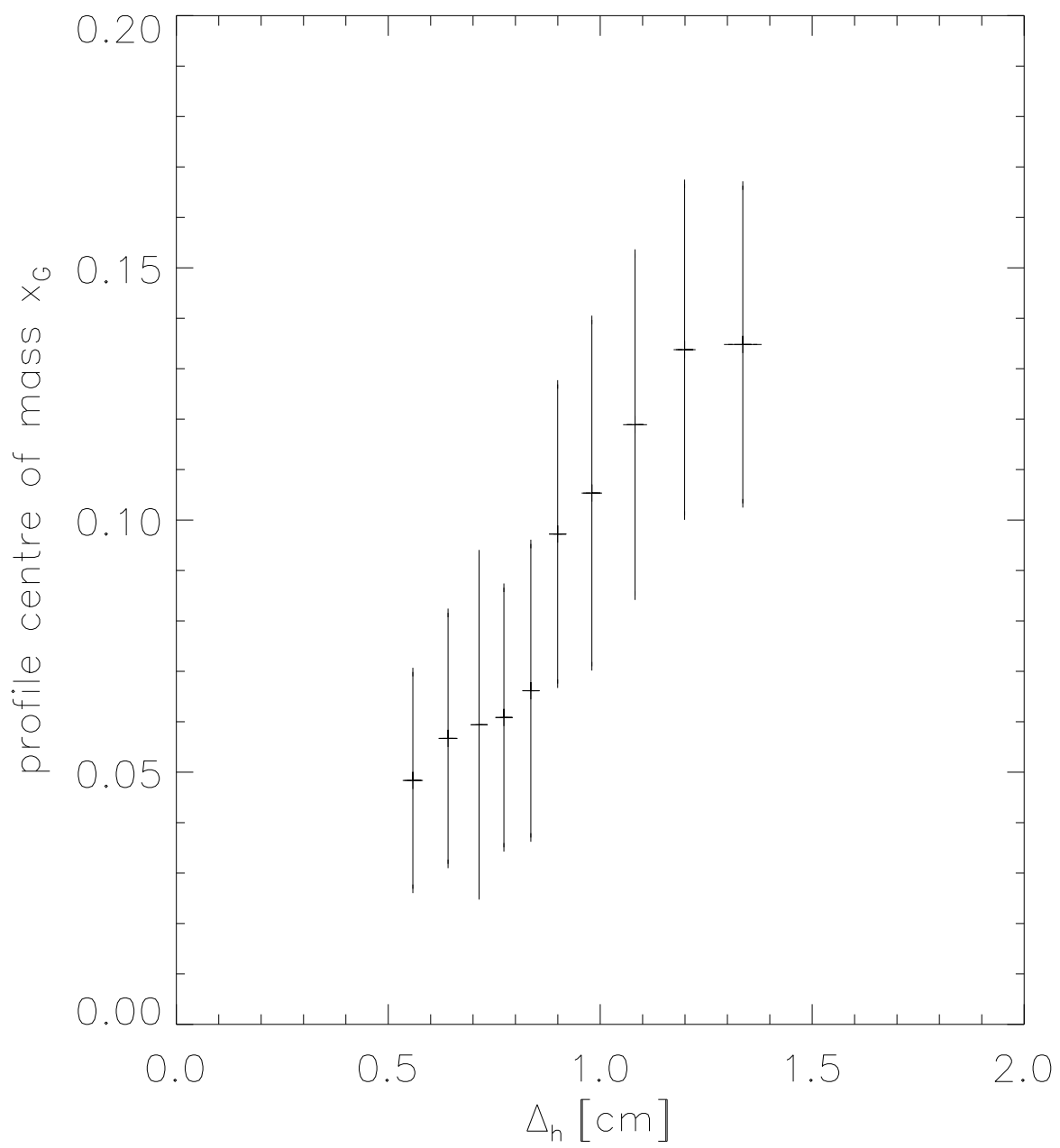

Figure 4: Normalized total radiation "centre of mass" $x_{g} / a$ versus magnetic horizontal shift $\Delta_{h}$. Each point is an ensamble average over many similar shots, and the error bars represent the spread of data around the average. 


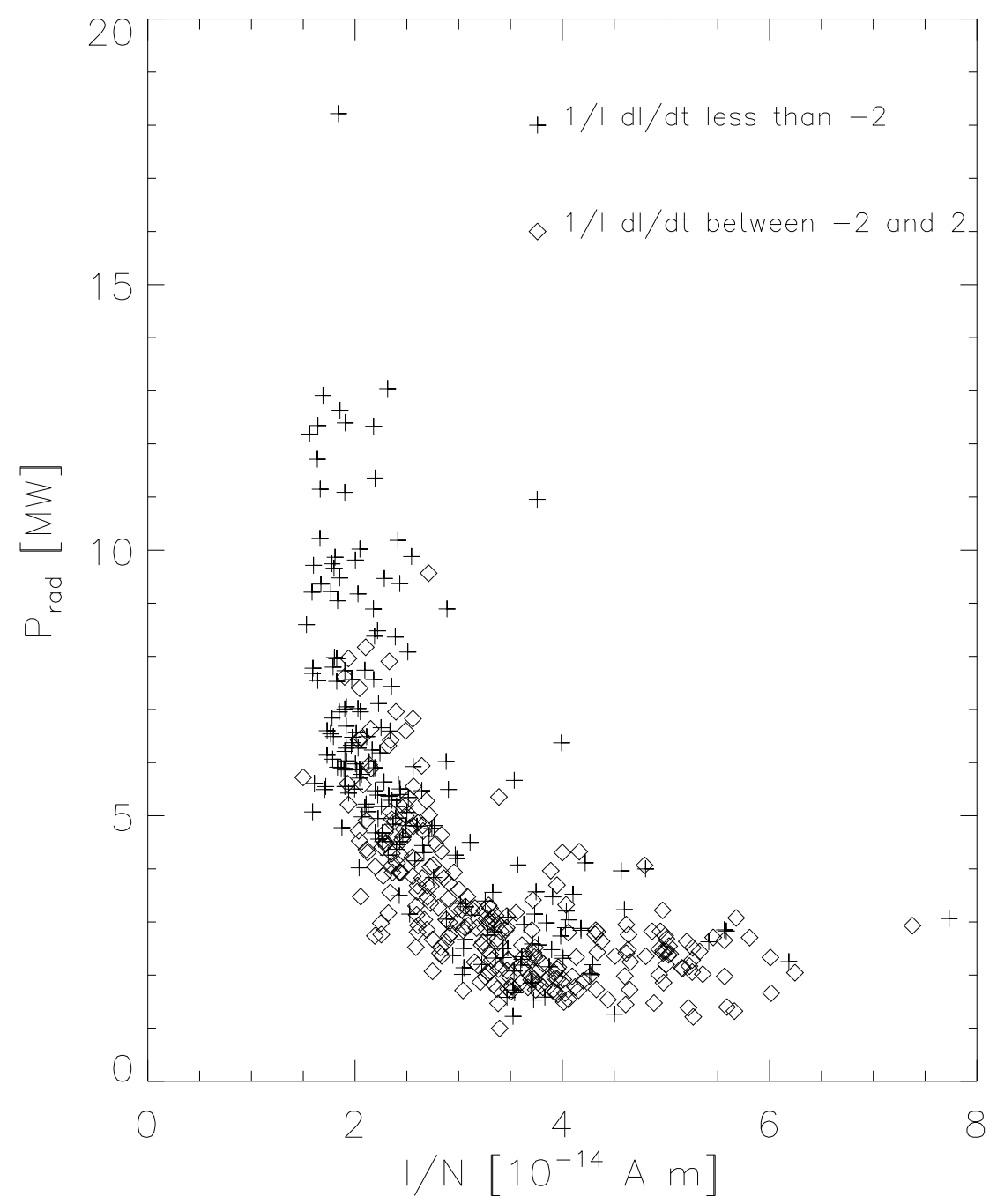

Figure 5: Total radiated power $P_{\text {rad }}$ vs $I / N$. Different symbols separate stationary from non-stationary discharges. 


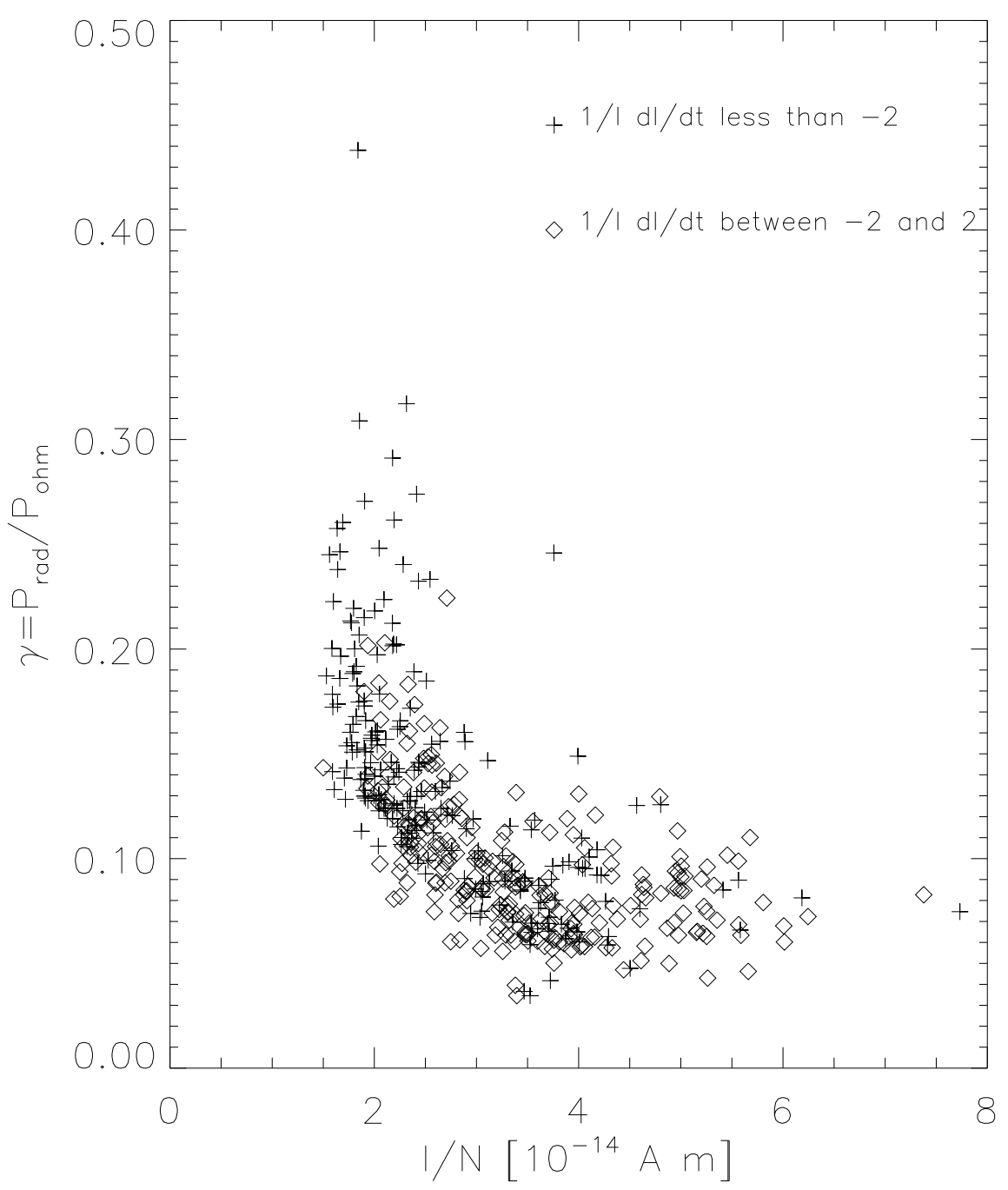

Figure 6: Ratio $\gamma=P_{\text {rad }} / P_{\text {ohm }}$ vs $I / N$. 


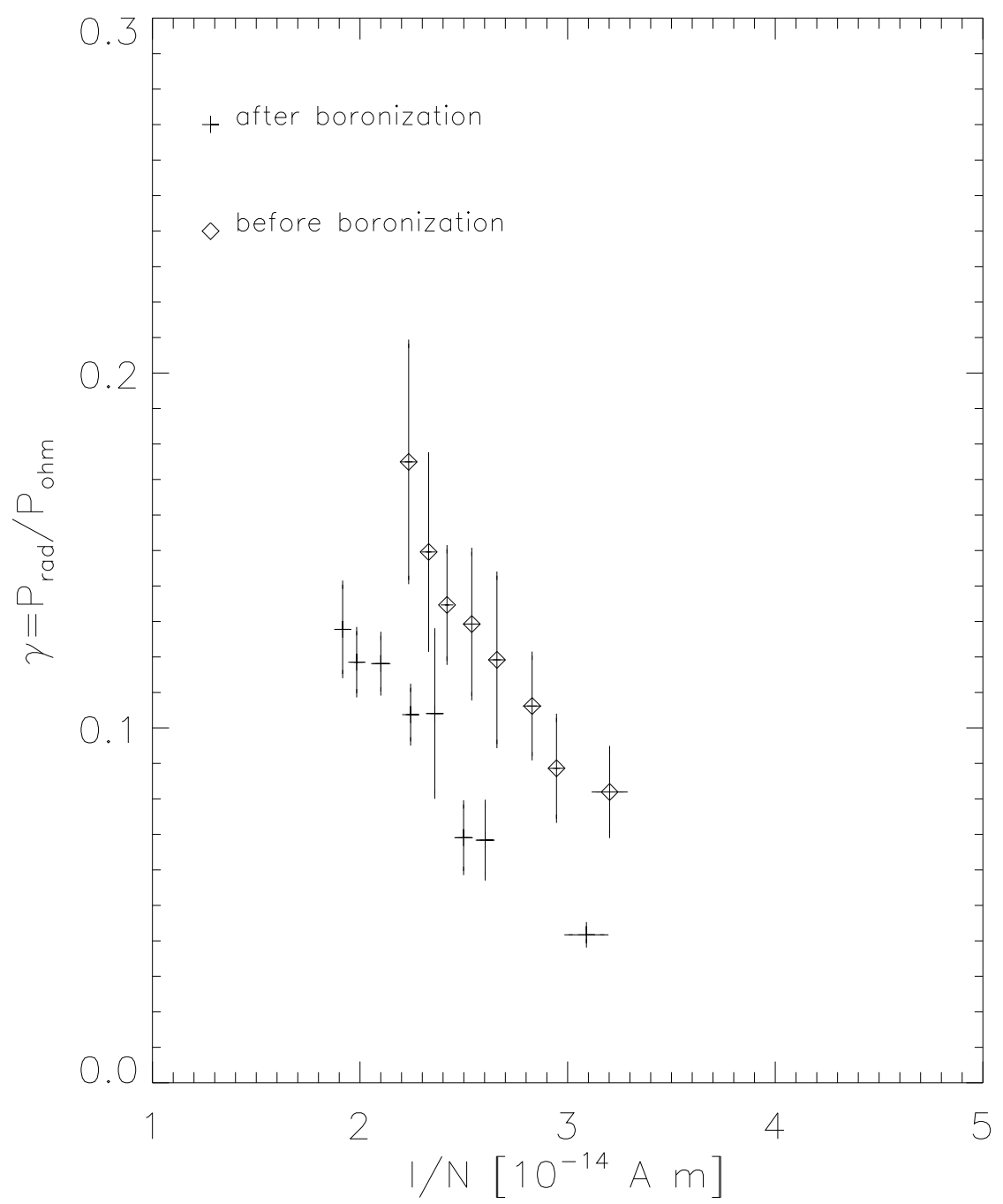

Figure 7: Ratio $\gamma=P_{\text {rad }} / P_{\text {ohm }}$ vs $I / N$, showing a comparison before and after wall conditioning with Boron: each point correspond to an ensamble average over many similar shots. 

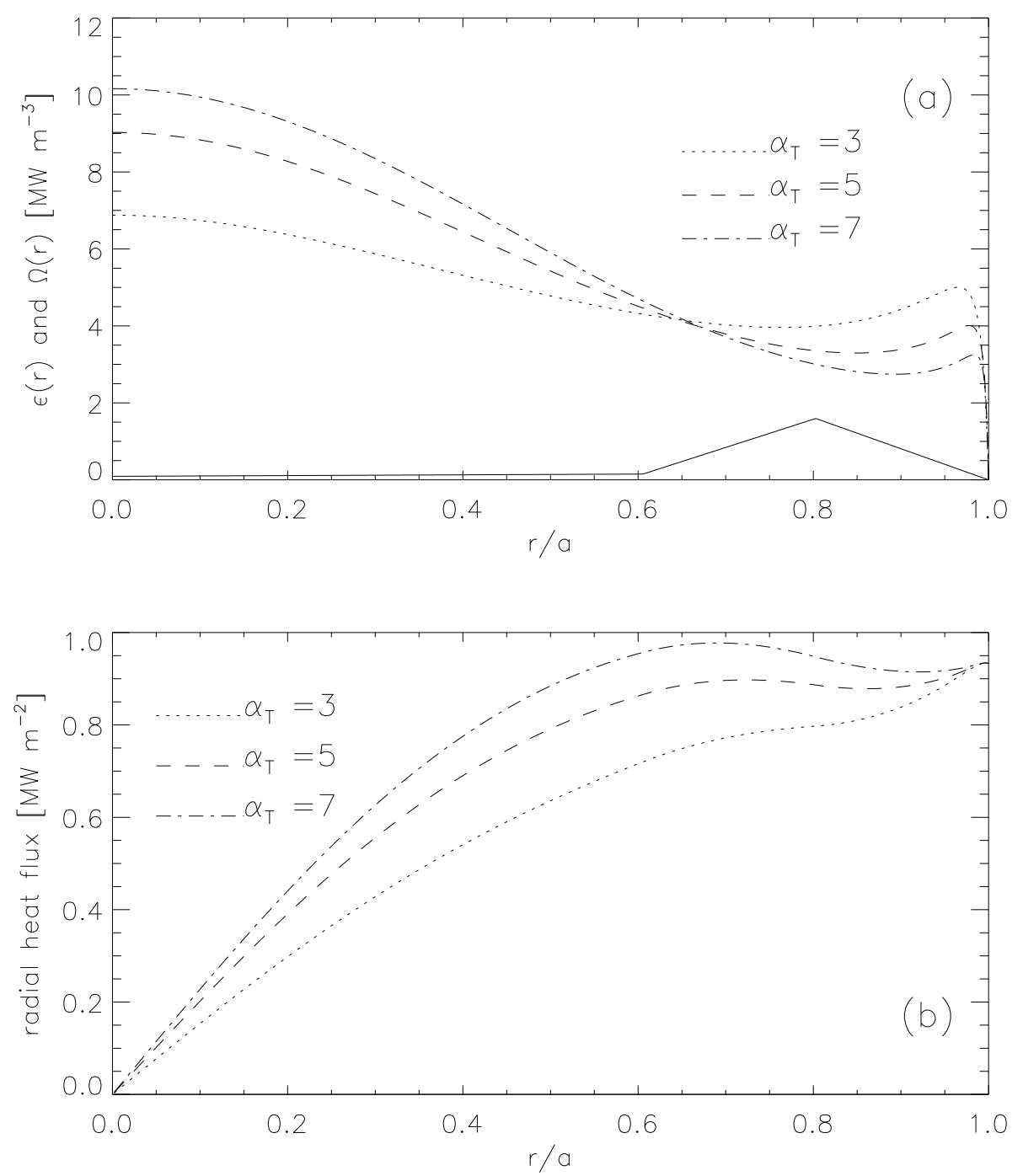

Figure 8: a) Emissivity profile (solid line) and local ohmic input power radial dependence for three different temperature profiles, characterized by $\alpha_{T}=3,5,7$. The data shown here corresponds to shot 4046, time $30 \mathrm{~ms}$, whose main parameters are: $I=0.85 \mathrm{MA}, n_{e}=5.3 \cdot 10^{19} \mathrm{~m}^{-3}, \gamma=13 \%, T_{e}(0)=320 \mathrm{eV}$. b) Radial dependence of the heat flux perpendicular to magnetic surfaces $q_{\perp}$, for the aforementioned temperature profiles. The $Z_{k}^{*}$ values change according to the different temperature profiles, giving $2.52,3.32$ and 3.72 , respectively. 